OPEN ACCESS

Edited by:

Caiji Gao,

South China Normal University, China

Reviewed by:

Qingqiu Gong,

Shanghai Jiao Tong University, China

Yonglun Zeng,

The Chinese University of Hong Kong,

China

*Correspondence:

Tong Su

sut0229@sdnu.edu.cn

Changle Ma

machangle@sdnu.edu.cn

${ }^{\dagger}$ These authors have contributed equally to this work

Specialty section:

This article was submitted to

Plant Cell Biology,

a section of the journal

Frontiers in Plant Science

Received: 07 August 2021

Accepted: 31 August 2021

Published: 23 September 2021

Citation:

Wang P, Wang T, Han J, Li M,

Zhao Y, Su T and Ma C (2021) Plant Autophagy: An Intricate Process Controlled by Various Signaling

Pathways.

Front. Plant Sci. 12:754982. doi: $10.3389 /$ fp/s.2021.754982

\section{Plant Autophagy: An Intricate Process Controlled by Various Signaling Pathways}

\author{
Pingping Wang ${ }^{\dagger}$, Tongtong Wang ${ }^{\dagger}$, Jingyi Han, Ming Li, Yanxiu Zhao, Tong Su* and \\ Changle Ma*
}

Shandong Provincial Key Laboratory of Plant Stress, College of Life Sciences, Shandong Normal University, Jinan, China

Autophagy is a ubiquitous process used widely across plant cells to degrade cellular material and is an important regulator of plant growth and various environmental stress responses in plants. The initiation and dynamics of autophagy in plant cells are precisely controlled according to the developmental stage of the plant and changes in the environment, which are transduced into intracellular signaling pathways. These signaling pathways often regulate autophagy by mediating TOR (Target of Rapamycin) kinase activity, an important regulator of autophagy initiation; however, some also act via TORindependent pathways. Under nutrient starvation, TOR activity is suppressed through glucose or ROS (reactive oxygen species) signaling, thereby promoting the initiation of autophagy. Under stresses, autophagy can be regulated by the regulatory networks connecting stresses, ROS and plant hormones, and in turn, autophagy regulates ROS levels and hormone signaling. This review focuses on the latest research progress in the mechanism of different external signals regulating autophagy.

Keywords: autophagy, signaling, nutrient, stress, plant hormone

\section{INTRODUCTION}

As immobile organisms, plants are subjected to various environmental stresses, such as nutrient deficiency, heat, salt, drought, cold, and pathogen infection (Zhu, 2016). Because these stresses can seriously impact normal plant growth and physiology, plants have evolved a complex set of regulatory mechanisms allowing them to adapt to adverse conditions. One particularly important response mechanism is the degradation of damaged or superfluous proteins and organelle structures through autophagy to maintain homeostasis of intracellular substances and to recycle nutrients (Marshall and Vierstra, 2018). Under normal conditions, low levels of constitutive autophagy activity are important for plant growth and development. Additionally, autophagy can be significantly induced to help plants resist adverse stress conditions (Wang et al., 2018a). Therefore, regulation of the timing and levels of autophagy are vital for plant survival.

Autophagy was first described by Christian de Duve in 1963 (Klionsky, 2008). It was not until the 1990s that the first autophagy gene Apg1 (Autophagy 1), later changed to autophagy-related gene ATG1, was identified in yeast (Matsuura et al., 1997). Since then, more than 30 ATGs have been identified in yeast (Wen and Klionsky, 2016). It is now thought that autophagy is present in all eukaryotes, sharing evolutionarily conserved core mechanisms (Liu and Bassham, 2012). Many homologs of yeast ATGs have been identified in plants (Hanaoka et al., 2002). In Arabidopsis, more than 40 ATGs have been found, most of which are homologs of yeast ATGs (Chung, 2019). Recently, 
great advances have been made in revealing the regulatory mechanisms of autophagy in plants and this review builds on recent research to present the latest understanding of the regulation of autophagy by various signaling pathways.

\section{THE MECHANISMS OF PLANT AUTOPHAGY}

Three forms of autophagy have been described in plants: microautophagy, macroautophagy and mega-autophagy (Marshall and Vierstra, 2018; Avin-Wittenberg, 2019; Gou et al., 2019; Figure 1). In microautophagy, cytosolic materials are gathered on the surface of the vacuole and directly captured through invagination of the tonoplast, forming membranewrapped autophagic bodies which will be degraded in the vacuole (Marshall and Vierstra, 2018). In contrast, macroautophagy is characterized by the formation of autophagosomes encapsulated in a bilayer membrane (Le Bars et al., 2014). A cup-shaped phagophore is formed at the phagophore assembly site (Zhuang et al., 2013). The membrane of the phagophore then elongates as it encircles cytosolic substances and closes off to form vesicular autophagosomes (Le Bars et al., 2014). The autophagosome is subsequently delivered to the vacuole (Ketelaar et al., 2004). The outer membrane of the autophagosome fuses with the tonoplast and the internal vesicle is released to the vacuolar lumen for degradation. Mega-autophagy is an extreme form of autophagy that so far has only been found in plants, and thus is not as well characterized as the main types of autophagy (Van Doorn and Papini, 2013). During mega-autophagy, the vacuolar membrane is permeabilized and ruptures, releasing large amounts of hydrolases into the cytoplasm, causing indiscriminate degradation of cytoplasmic materials (Hatsugai et al., 2004; Van Doorn and Papini, 2013). Mega-autophagy occurs in plants during developmental PCD (programmed cell death), which is a result of normal development as well as abiotic stresses, but does not occur in pathogen-induced PCD. Additionally, mega-autophagy leads to cell death rather than cell remobilization or stress resistance. Distinct from canonical autophagy, mega-autophagy does not involve capture of cellular components into the vacuole. No ATG genes have been reported to be involved in mega-autophagy. Therefore, mega-autophagy is often referred to as autolysis.

The underlying mechanism of macroautophagy (autophagy for short) has been more clearly characterized than that of microautophagy. A series of ATG proteins mediate the entire process of macroautophagy. The initiation of autophagy can be regulated by an essential serine/threonine kinase, TOR (Target of Rapamycin), which negatively regulates autophagy (DíazTroya et al., 2008). TOR activity is regulated by nutritional signals and environmental stresses (Dobrenel et al., 2016; Fu et al., 2020). Under normal conditions, Arabidopsis TOR is active and maintains the hyperphosphorylated state of its substrate, ATG13, thereby inhibiting the binding of ATG13 to ATG1 (Suttangkakul et al., 2011). Under nutrient-deficient conditions, TOR becomes inactivated, resulting in the dephosphorylation of ATG13, which triggers interaction between ATG13 and ATG1.
ATG1-ATG13 then interact with two other subunits, ATG11 and ATG101, to form a complex which mediates autophagy initiation. The ATG1-ATG13-ATG11-ATG101 complex activates the subsequent steps of autophagy, including vesicle nucleation and phagophore expansion and closure (Suttangkakul et al., 2011; Li and Vierstra, 2014). The ATG9-ATG2-ATG18 complex is responsible for autophagosome formation and phagophore expansion via delivery of additional lipids (Yamamoto et al., 2012; Zhuang et al., 2017; Kang et al., 2018). Simultaneously, ATG8phosphatidylethanolamine (PE) is inserted into the membrane of the expanding phagophore via a ubiquitin-like conjugation pathway (Ohsumi, 2001; Minina et al., 2018). Immature ATG8 precursors are transformed to mature ATG8 by the cysteine protease ATG4, and then are activated by ATP-dependent ubiquitin-activating enzyme (E1)-like ATG7 (Yoshimoto et al., 2004; Woo et al., 2014; Minina et al., 2018). ATG8 is subsequently delivered to the ubiquitin-conjugating enzyme (E2)-like ATG3 and finally conjugated to PE by a complex of ubiquitin-ligase enzymes (E3)-like ATG12-ATG5-ATG16 (Chung et al., 2010). ATG8 is regarded as a marker of the autophagosome and plays a vital role in the specific recognition of autophagy cargoes through the interaction of ATG8 with autophagy receptors (Liu and Bassham, 2012). The outer membrane of the autophagosome finally fuses with the vacuolar membrane and releases the cargoes into the vacuolar lumen for degradation (Liu and Bassham, 2012).

\section{ACTIVATION OF AUTOPHAGY THROUGH THE TOR SIGNALING PATHWAY}

TOR belongs to the phosphatidylinositol 3 kinase (PI3K)related kinase family and can form two distinct complexes, TORC1 and TORC2 (TOR complex 1 and 2) in yeast and mammals. Among these, TORC1 regulates protein synthesis and the initiation of autophagy (Díaz-Troya et al., 2008). The TORC1 complex also exists in plants and algae (Mahfouz et al., 2006; Shemi et al., 2015). In plants, TORC1 is consist of a catalytic subunit TOR, a regulatory subunit LST8 (lethal with sec thirteen 8), and RAPTOR (regulatory-associated protein of TOR). The Arabidopsis RAPTOR family, consisting of RAPTOR1A and RAPTOR1B, functions as the target recognition cofactor of TOR (Anderson et al., 2005; Deprost et al., 2005). It is demonstrated that RAPTOR1B is involved in TOR-mediated autophagy repression ( $\mathrm{Pu}$ et al., 2017). Two putative homologs of LST8, LST8-1 and LST8-2, have been identified in Arabidopsis (Moreau et al., 2012), and LST8-1 participates in adaptation to long days and hormone signaling (Moreau et al., 2012; Kravchenko et al., 2015). In plants, the TOR complex regulates the initiation of autophagy by modulating the ATG1-ATG13 complex in response to stresses (Mugume et al., 2020). There are four ATG1 isoforms and two ATG13 isoforms encoded in the Arabidopsis genome (Huang et al., 2019b). Both ATG1 and ATG13 are reversibly phosphorylated by TOR (Van Leene et al., 2019). ATG13 contains a canonical TOS (TOR signaling) motif, which mediates the interaction of ATG13 with RAPTOR (Son et al., 2018). During nutrient starvation, ATGla is hyperphosphorylated by 


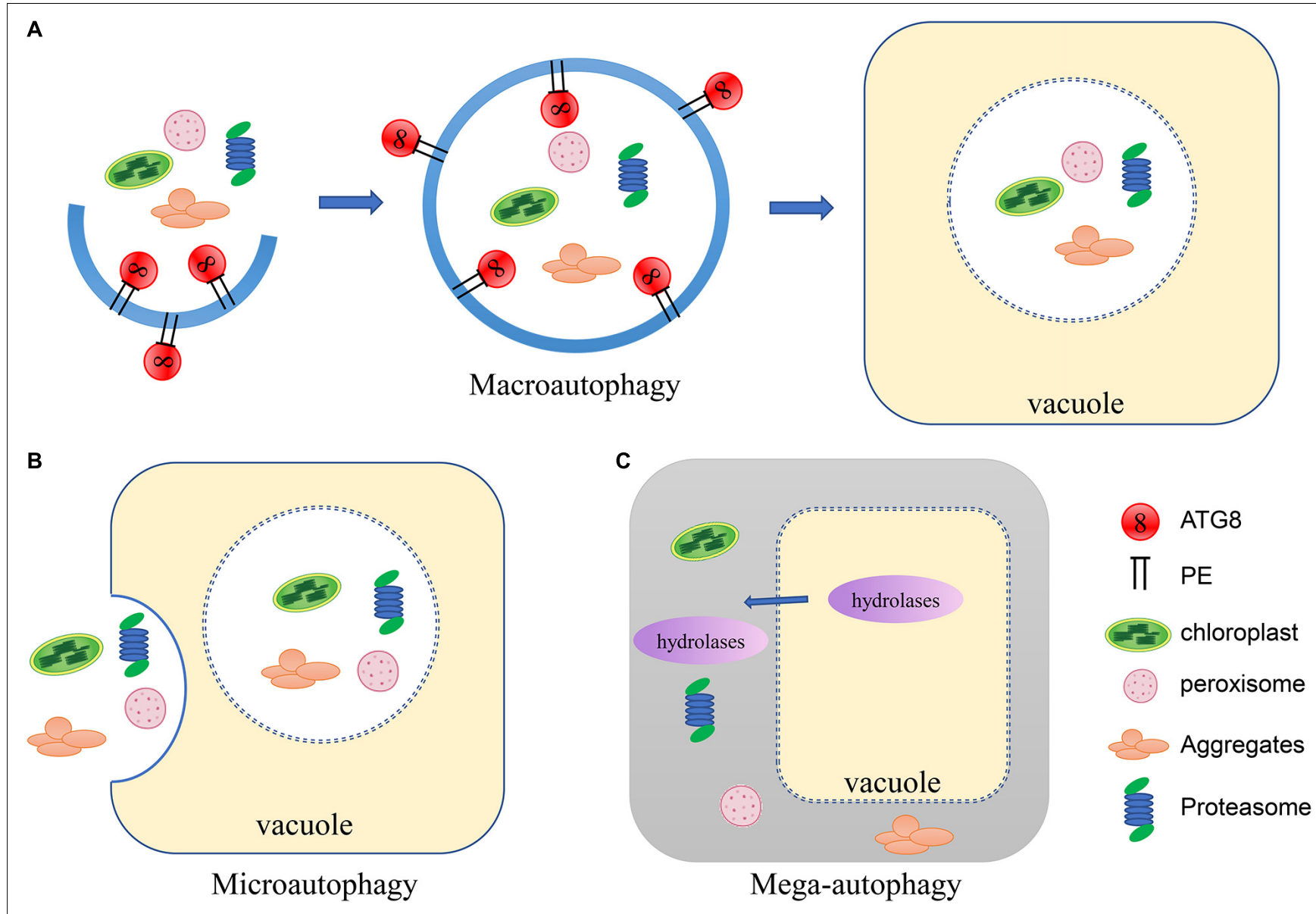

FIGURE 1 | Schematic of the three types of autophagy in plants. (A) In macroautophagy, a cup-shaped phagophore is formed and closes into vesicular autophagosomes as it encircles cytosolic substances. The autophagosome is subsequently delivered to the vacuole. The outer membrane of the autophagosome fuses with the tonoplast and the internal vesicle is released to the vacuolar lumen for degradation. (B) In microautophagy, cytosolic materials are gathered on the tonoplast and directly captured by the vacuole through invagination to be degraded. (C) During mega-autophagy, the vacuolar membrane is permeabilized and ruptures, releasing large amounts of hydrolases into the cytoplasm, causing indiscriminate degradation of cytoplasmic materials.

the SnRK1 (sucrose non-fermentation1-related protein kinase 1) complex and ATG13a is dephosphorylated (Suttangkakul et al., 2011; Chen et al., 2017; Soto-Burgos and Bassham, 2017). The hyperphosphorylated ATG1 interacts with dephosphorylated ATG13 to form an active complex along with ATG11 and ATG101 to initiate autophagy (Suttangkakul et al., 2011; Li and Vierstra, 2014).

\section{REGULATION OF AUTOPHAGY BY NUTRIENT SIGNALS}

Under nutrient starvation, one of the most effective ways for plants to adapt is to degrade excess cytoplasmic material through autophagy to allow nutrients to be reused (Masclaux-Daubresse et al., 2014). Arabidopsis atg (atg5, atg7, and atg18) mutants show more severe chlorosis and reduced survival rates compared with wild type under nitrogen deprivation conditions (Thompson et al., 2005; Xiong et al., 2005). N remobilization from the leaves into the seeds has been evaluated using the isotopic tracer method, wherein plants are fed with ${ }^{15} \mathrm{NO}_{3}$ and subsequently ${ }^{15} \mathrm{~N}$ is detected in the seeds (Guiboileau et al., 2012). Under N-limited conditions, ${ }^{15} \mathrm{~N}$ remobilization is sharply decreased in atg5, atg9, and $\operatorname{atg} 18 a$ mutants compared with wild type (Guiboileau et al., 2012). Salicylic acid (SA) is accumulated in atg mutants and is responsible for the early leaf senescence phenotype of atg mutants. However, the ${ }^{15} \mathrm{~N}$-remobilization defect was not related to the early leaf senescence phenotype of the atg mutants (Guiboileau et al., 2013). Correspondingly, the rosette leaves of the atg mutants contained more ammonium, amino acids, and proteins but less sugars and starch compared to wild type, indicating that autophagy may affect the $\mathrm{C} / \mathrm{N}$ balance of plants (Guiboileau et al., 2013; Izumi et al., 2013). The free amino acid content in atg mutants is increased under nitrogen starvation, consistent with their observed nitrogen remobilization defect (Guiboileau et al., 2013; Masclaux-Daubresse et al., 2014).

Autophagy also plays an important role under short day conditions. In Arabidopsis, compared with the starchless mutant pgm (phosphoglucomutase), the double mutant pgm atg5 displays early cell death in leaves under short day conditions and reduced 
production of free amino acids during the night, suggesting that autophagy regulates the production of free amino acids which can act as an energy source during carbon starvation (Izumi et al., 2013). In addition, Arabidopsis atg 5 and atg 7 etiolated seedlings exhibit shorter hypocotyls and produce fewer free amino acids compared with wild type seedlings when grow on fixed-carbon medium, indicating that autophagy has wide influences on metabolism during carbon starvation (Avin-Wittenberg et al., 2015). During prolonged carbon starvation, but not under nitrogen deprivation, Arabidopsis ATG6 can be phosphorylated by SnRK1 to initiate autophagy via an ATG1-independent autophagy initiation pathway, indicating that the initiation of autophagy may be regulated by different mechanisms in response to nitrogen and carbon starvation (Huang et al., 2019b).

After sulfur is absorbed by plants in the form of sulfate, it is successively converted into sulfide by various enzymes, including ATP sulfurylase (ATPS), APS reductase (APR), and sulfite reductase (SIR) (Takahashi et al., 2011). The mutant sir1-1 displays inhibited TOR activity and reduced glucose levels when grown in sulfate deprivation conditions (Dong et al., 2017). TOR activity in sir1-1 can be restored by exogenous glucose, indicating that sulfur may regulate TOR activity through glucose signaling (Dong et al., 2017). In Arabidopsis, glucose may activate TOR by inhibiting the activity of SnRK1 (Baena-González and Hanson, 2017). SnRK1 directly phosphorylates RAPTOR1B, thus inhibiting TOR activity (Nukarinen et al., 2016). Alternatively, sulfur starvation may directly promote autophagy through other unknown pathways.

In Arabidopsis, D-glucose levels are sensed by heterotrimeric G protein complex and RGS1 (regulator of G-protein signaling 1), a member of a glucose-sensing complex (Chen and Jones, 2004). Turnover of the RGS1 protein is crucial for cell cycle regulation and the cellular glucose signaling pathway (Jiao et al., 2019). In Arabidopsis, the expressions of ATGs are much more induced in wild type than in rgs 1 when treated with $1 \%$ glucose, indicating that RGS1 promotes autophagy (Yan et al., 2017). Furthermore, it was demonstrated that proteasome-independent degradation of RGS1, as well as autophagic flux, can be induced by nutrient starvation. RGS1 directly interacts with ATG8 and co-localizes with autophagosomes in Arabidopsis root cells, indicating that autophagy mediates the degradation of RGS1 in response to sugar signaling (Jiao et al., 2019).

It has been reported that ER stress-dependent autophagy can be rapidly activated in plant roots in response to phosphate (Pi) starvation (Naumann et al., 2019). Arabidopsis LPR1 (LOW PHOSPHATE RESPONSE 1) encodes a cell wall-targeted ferroxidase (Müller et al., 2015), which is involved in deposition of callose and pectin polymers in cell walls, inhibition of cell-tocell communication and maintenance of the root apical meristem under Pi deficiency conditions (Hoehenwarter et al., 2016). LPR1 activity can be inhibited by PDR2 (PHOSPHATE DEFICIENCY RESPONSE2), the ER-resident AtP5A, which is involved in the ER stress response (Ticconi et al., 2009; Müller et al., 2015; Sørensen et al., 2015). Thus, local root Pi sensing and ER stressdependent autophagy initiation are connected by the PDR2-LPR1 module (Naumann et al., 2019). It has been reported that the
S-domain receptor kinase (ARK2) and $U$ box/Armadillo repeatcontaining E3 ligase (PUB9) regulate lateral root growth under Pi deficiency conditions in Arabidopsis (Sankaranarayanan and Samuel, 2015). During Pi starvation, activation of ARK2 results in phosphorylation of PUB9, which is likely involved in the selective degradation of AUX/IAA (Auxin/Indole-3-Acetic Acid) protein or other repressors of auxin accumulation via autophagy. This leads to an increase of auxin in lateral root initiation sites (Sankaranarayanan and Samuel, 2015).

Over-induction of autophagy can impair the normal functions of plant cells, particularly during prolonged starvation or during nutrient recovery after starvation. Therefore, it is sometimes necessary to inhibit autophagy to ensure that it is maintained at a reasonable level. It was reported that Arabidopsis RING-type E3 ligases SINAT1 (SEVEN IN ABSENTIA OF ARABIDOPSIS THALIANA1), SINAT2, and SINAT6 regulate ATG1 and ATG13 levels via ubiquitination of ATG13, thereby regulating the dynamics of autophagy (Qi et al., 2019). During nutrient-rich conditions, ATG13 is ubiquitinated by the SINAT1-SINAT2TRAF1a-TRAF1b (TUMOR NECROSIS FACTOR RECEPTOR ASSOCIATED FACTOR) complex and degraded via the ubiquitin proteasome pathway, thus maintaining low levels of autophagy (Qi et al., 2019). Under prolonged nutrient starvation, ATG13 is similarly degraded through the ubiquitin proteasome pathway, mediated by SINAT1 and SINAT2, to downregulate autophagy (Qi et al., 2019). In contrast, SINAT6 has an opposite effect, whereby it competitively interacts with ATG13 to inhibit the ubiquitination and degradation of the ATG1/ATG13 complex, leading to activation of autophagy during nutrient starvation (Qi et al., 2019). These results suggest that regulation of autophagy by various intracellular signals is a very delicate process, which ensures that plants can respond to environmental changes in an appropriate and timely manner (Figure 2).

\section{REGULATION OF AUTOPHAGY BY BIOTIC STRESS SIGNALS}

Plants often suffer from infection by pathogens or plant viruses, which can stimulate the host innate immunity of plants. As the signal source, the pathogen can be recognized by membrane-localized or cytoplasmically-localized receptors, thereby triggering an immune response (Peng et al., 2018). Autophagy is induced by many pathogens' recognition receptors to directly target intracellular pathogens for selective degradation (Deretic, 2012). In Nicotiana benthamiana, Rice stripe virus (RSV) infection is promoted in $\operatorname{atg} 5$ or $\operatorname{atg} 7$ mutants. It has been shown that NbP3IP can interact with p3, an RNA silencing suppressor protein encoded by RSV, to mediate the autophagic degradation of p3 and suppression of the RSV infection (Jiang et al., 2021a). NbP3IP is a potential selective autophagy cargo receptor and can interact with NbATG8f. Similarly, the rice homolog OsP3IP also mediates p3 degradation via interaction with OsATG8b and p3 (Jiang et al., 2021a). When CFP-NbATG8f was co-expressed with NbP3IP-Myc in Nicotiana benthamiana, an increase in CFP labeled autophagic structures was detected, indicating that P3IP participates in the regulation of autophagy during the plant viral 


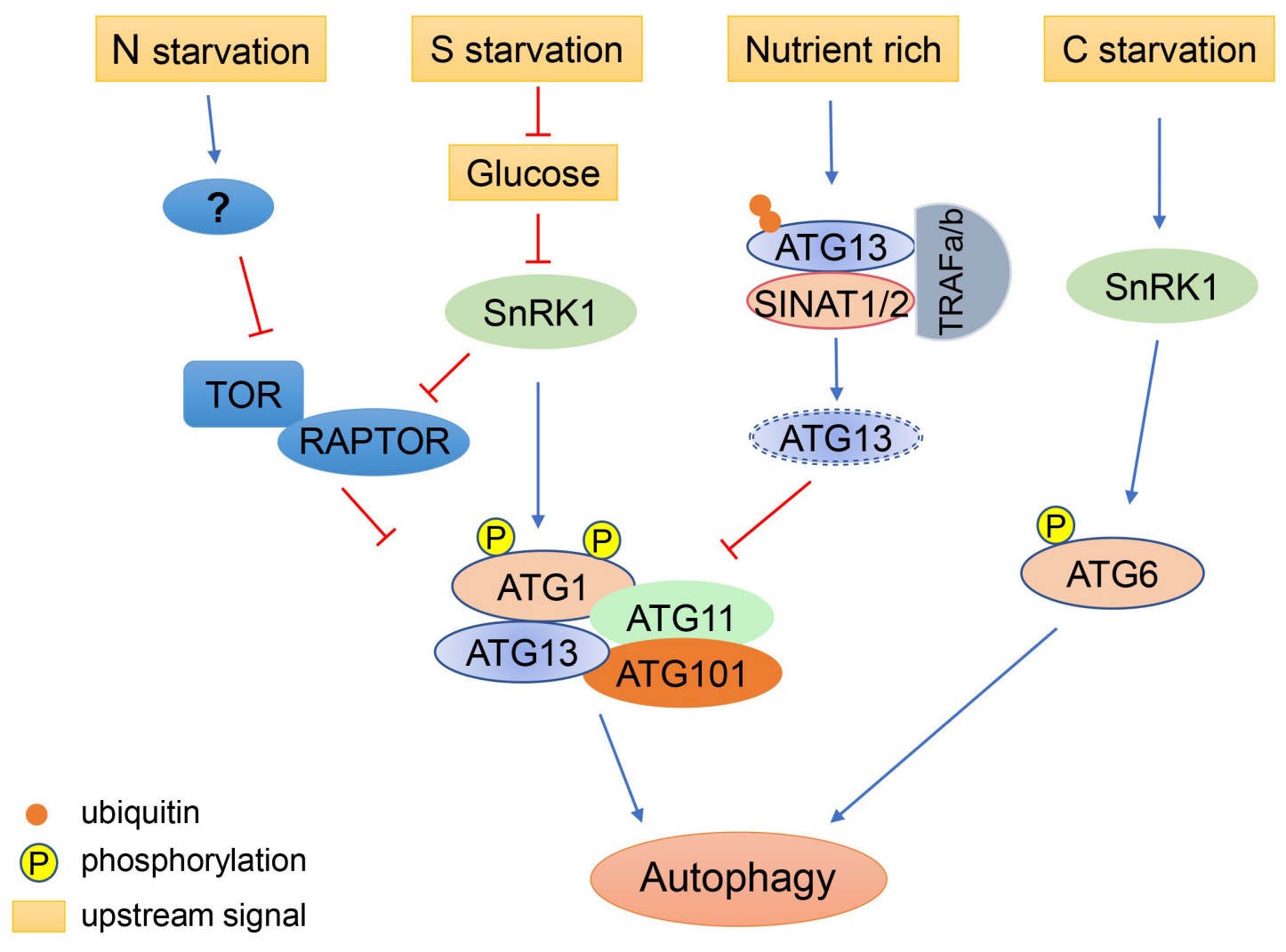

FIGURE 2 | Regulation of autophagy initiation under different nutritional conditions. Autophagy is induced by nitrogen (N) starvation in plants through inhibition of TOR. Sulfur (S) starvation regulates autophagy through the repression of glucose signaling, resulting in the activation of SnRK1, which inhibits TOR and activates the ATG1 autophagy initiation complex. Under nutrient-rich conditions, ATG13 is ubiquitinated by the SINATs-TRAF1s complex and degraded via the proteasome pathway, maintaining low levels of autophagy. Under prolonged carbon (C) starvation, ATG6 can be phosphorylated by SnRK1 to initiate autophagy via an ATG1-independent pathway.

defense response (Jiang et al., 2021b). In Arabidopsis, a small 71 amino acid peptide, VISP1, can be induced by Cucumber mosaic virus (CMV) infection and interacts with ATG8 through UIM (ubiquitin-interacting motif) motif. VISP1 functions as a selective autophagy receptor involved in degradation of SGS3 (suppressor of gene silencing 3)/RDR6 (RNA-dependent RNA polymerase 6)-bodies to negatively regulate siRNA amplification, resulting in suppression of antiviral immunity (Tong et al., 2021).

Autophagy can also prevent the spread of pathogen infection by promoting hypersensitive response programmed cell death (HR-PCD) (Zhou et al., 2014). Autophagy-deficient Arabidopsis atg7 and atg9 mutants show a weakened PCD response after infection with Pseudomonas syringae pv. tomato DC3000 harboring the avirulence gene AvrRPS4 (Hofius et al., 2009). Thus, in this case, autophagy limits the spread of the pathogen by promoting cell death. But on the other hand, during the immune response of plants to avirulent biotrophic pathogens and necrotrophic pathogens, autophagy can restrict the spread of unnecessary cell death to promote cell survival (Teh and Hofius, 2014; Zhou et al., 2014). HR-PCD is induced at the site of infection when wild type Nicotiana benthamiana leaves are infected with Phytophthora infestans or Pseudomonas syringae pv. tomato DC3000. In Nicotiana benthamiana autophagydeficient plants, HR-PCD spreads uncontrollably to uninfected cells (Liu et al., 2005). In Arabidopsis, Psm ES4326/AvrRpt2 can trigger autophagy-dependent HR via the EDS1 (enhanced disease susceptibility 1) pathway, but NPR1 (non-expressor of pathogenesis-related genes 1) suppresses the autophagy flux (Gong et al., 2020). In Arabidopsis atg $4 a 4 b$ double mutant, total SA (salicylic acid) content decreased and free SA unchanged compared with wild type after AvrRpt2 infection, indicating that ATG4 can suppress the consumption of free SA. ATG4 inhibits SA consumption resulting in the inhibition of NPR3 (non-expressor of pathogenesis-related genes 3) synthesis and enhanced expression of NPR1, which inhibits autophagy during AvrRpt2 affection. These results indicate that ATG4 mediates AvrRpt2-induced autophagy dependent on SA (Gong et al., 2020). Therefore, the role of autophagy in the immune response depends on what pathogen signals the plant cell receives.

Furthermore, some pathogens can even facilitate their infection in plants by regulating autophagy. The $\gamma \mathrm{b}$ protein from Barley stripe mosaic virus can directly interacts with Nicotiana benthamiana ATG7 to interfere with the interaction of ATG7 with ATG8f in a competitive manner, resulting 
in the suppression of autophagy (Yang et al., 2018). In Arabidopsis, TOR activity is inhibited by the effector AWR5 (alanine-tryptophan-arginine tryad) secreted from the bacterium Ralstonia solanacearum to help feed the pathogen through autophagy induction (Popa et al., 2016). Additionally, autophagy can be induced by activation of salicylic acid signaling in a NPR1-dependent manner when Arabidopsis is infected with P. syringae (Yoshimoto et al., 2009). Moreover, a type III effector (T3E), Hrp outer protein M1 (HopM1), secreted by $P$. syringae can interact with proteasomes to promote the selective degradation of proteasomes via autophagy, inhibiting salicylic acid-dependent defense responses (Üstün et al., 2018). However, NBR1 (NEIGHBOR OF BRCA1)-dependent selective autophagy prevents the formation of HopM1-mediated water-soaked lesions and thereby dampens bacterial virulence and proliferation (Üstün et al., 2018). In summary, the roles of autophagy in the plant immune system are the result of interactions between various signaling pathways (Figure 3).

\section{INDUCTION OF AUTOPHAGY BY ABIOTIC STRESS SIGNALS}

Autophagy can be induced by various abiotic stresses, including heat, cold, drought, osmotic, and salt stress, to improve plant resistance (Slavikova et al., 2008; Dündar et al., 2019; Sedaghatmehr et al., 2019; Bao et al., 2020; Chi et al., 2020; Jung et al., 2020; Thirumalaikumar et al., 2021). TOR kinase, which is crucial for autophagy induction, works downstream of SnRK1 (Soto-Burgos and Bassham, 2017). In response to abiotic stress, Arabidopsis trehalose 6-phosphate (T6P) suppresses SnRK1 activity, which may result in inhibition of autophagy

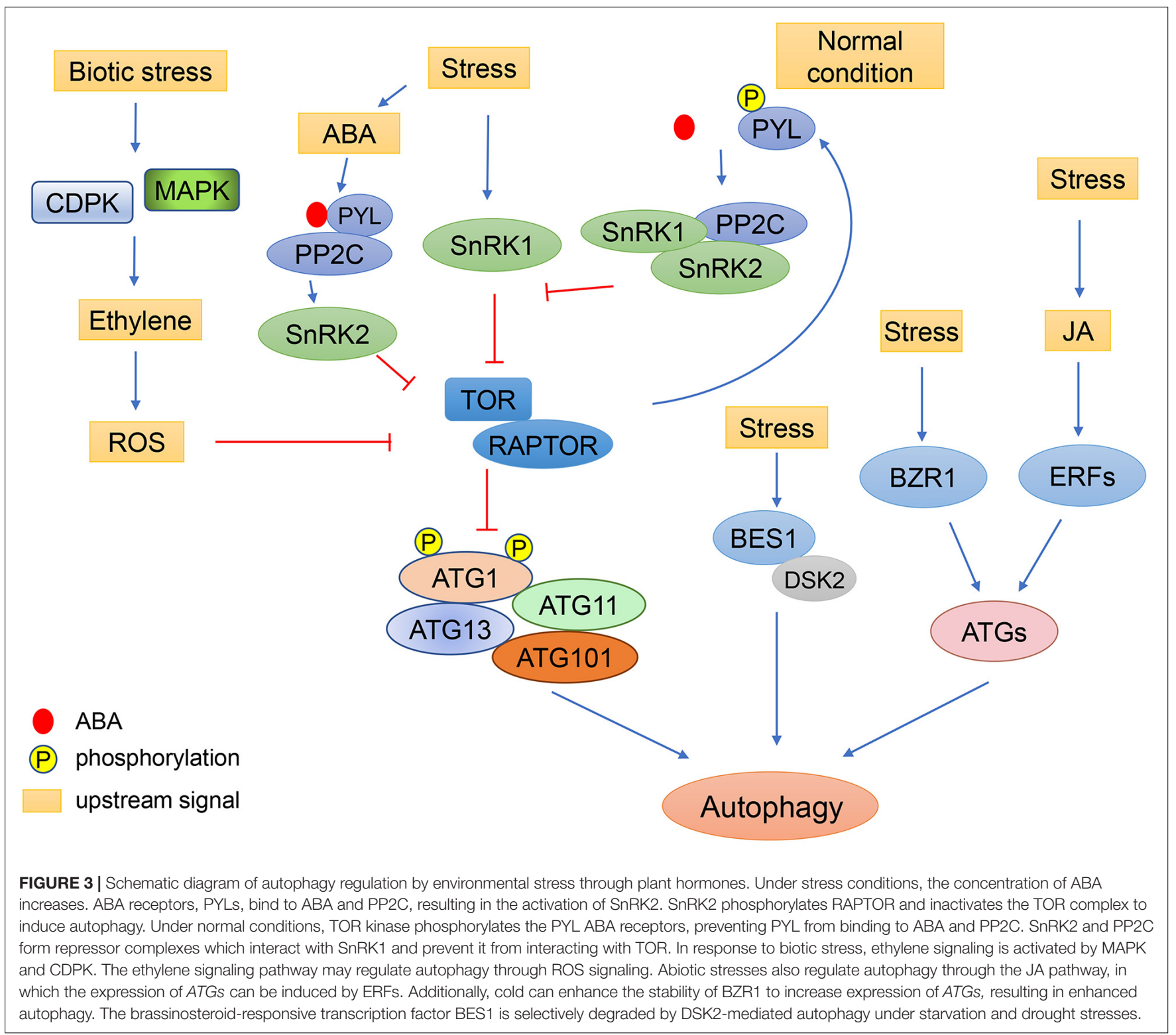


(Tsai and Gazzarrini, 2014; Soto-Burgos and Bassham, 2017). SnRK1 inhibits the activity of TOR by phosphorylating RAPTOR1B. In Arabidopsis, KIN10, a SnRK1 catalytic subunit, can also phosphorylate RAPTOR1B (Nukarinen et al., 2016). Overexpression of KIN10 increases autophagy, whereas knockout of KIN10 inhibits abiotic stress-induced autophagy in Arabidopsis. SnRK1 can also regulate autophagy in a TORindependent manner, during which the ATG1 complex can be phosphorylated and activated directly by SnRK1 to induce autophagy (Chen et al., 2017; Soto-Burgos and Bassham, 2017).

The protein phosphatase 2A-associated protein (PP2Aassociated protein, TAP46), a regulatory subunit of PP2A, can be phosphorylated by TOR kinase, inducing PP2A activity to inhibit the formation of the double-membraned autophagosomes (Máthé et al., 2021). Silencing of TAP46 activates autophagy in tobacco and Arabidopsis, and the tap46 mutant shows the same phenotype as tor mutants, including TOR inactivation resulting in induction activation of autophagy and nitrogen mobilization, indicating that TAP46 negatively regulates autophagy via TOR signaling (Ahn et al., 2011).

Plants can also regulate autophagy independently of TOR kinases. Under carbon starvation, Arabidopsis KIN10 phosphorylates ATG6 to activate the PI3K complex and initiate autophagy (Huang et al., 2019b). Under oxidative stress and ER stress, KIN10 can induce autophagy by directly activating the ATG1 complex (Chen et al., 2017). Levels of phosphorylated ATG1 are increased by overexpression of KIN1, and KIN10 can interact with ATG1a and ATG13a in vitro, indicating that KIN10 most likely phosphorylates ATG1 in TOR-independent autophagy (Chen et al., 2017; Figure 3).

ATG8, a marker of the autophagosome, is involved in the specific recognition of autophagy cargoes. In plants, ATG8 also plays an important role in the regulation of autophagy in response to abiotic stresses. In Arabidopsis, a plant-specific developmental regulatory protein COST1 (constitutively stressed 1) directly interacts with ATG8e to inhibit autophagy under normal conditions to promote plant growth. Under drought stress, COST1 is degraded via the ubiquitin proteasome system (UPS) and autophagy, releasing ATG8 to promote autophagosome formation and drought tolerance (Bao et al., 2020).

The expression of multi-stress regulatory genes which encode plant tryptophan-rich sensory protein (TSPO) can be induced by osmotic stress and salt stress (Guillaumot et al., 2009). AtTSPO directly binds to ATG8 through the AIM domain of AtTSPO and also interacts with a plasma membrane aquaporin, PIP2;7, to mediate the autophagic degradation of PIP2;7, which subsequently reduces intercellular water transport under water deficient conditions (Vanhee et al., 2011; Hachez et al., 2014). The degradation of PIP2;7 can be triggered by abscisic acid (ABA) treatment, indicating that $\mathrm{ABA}$ may participate in selective autophagy in response to osmotic stress (Hachez et al., 2014). Two plant specific proteins, ATI1 and its homolog ATI2, can also interact with ATG8 via putative AIM motifs during salt stress to mediate the degradation of chloroplast membranes and fragments via autophagy, indicating that the selective degradation mediated by ATI1 and ATI2 is involved in salt tolerance in plants (Honig et al., 2012; Michaeli et al., 2014). In Arabidopsis, ATI1 and ATI2 are also involved in the regulation of reticulophagy induced by starvation stress during darkness (Wu et al., 2021). Under carbon starvation, ATI1 and ATI2 interact with ERlocalized MSBP1 (Membrane Steroid Binding Protein 1) to mediate the autophagic degradation of MSBP1, indicating that ATI1 and ATI2 serve as MSBP1 cargo receptors to regulate the selective reticulophagy during starvation stress (Wu et al., 2021).

\section{THE INTERACTION BETWEEN HORMONE SIGNALING AND AUTOPHAGY}

Phytohormones play comprehensive and vital roles during plant growth and development under normal and adverse conditions (Gou et al., 2019). Recently, considerable progress has been made to uncover the crosstalk between autophagy and plant hormone signals (Figure 3).

$\mathrm{ABA}$ is an essential endogenous signal which acts during various stresses in plants to regulate leaf expansion and stomatal closure (Raghavendra et al., 2010). Under osmotic stresses such as drought and high salinity, ABA accumulates in all plant organs, indicating ABA is a systemic signaling molecule (Yoshida et al., 2014). Under stress conditions, TOR activity can be inhibited by ABA signaling to induce autophagy (Wang et al., 2018b). SnRK2, as a kinase downstream of ABA, can phosphorylate RAPTOR and inactivate the TOR complex (Wang et al., 2018b). But under normal conditions, TOR kinase phosphorylates PYL (pyrabactin resistance1/PYR1-like) ABA receptors, preventing the PYL receptors from binding to $\mathrm{ABA}$ and $\mathrm{PP} 2 \mathrm{C}$ phosphatase effectors, resulting in the inactivation of SnRK2 kinases (Wang et al., 2018b). In the absence of ABA, SnRK2 and PP2C are involved in the formation of a repressor complex which interacts with SnRK1 and prevents it from interacting with TOR. However, in the presence of ABA, this repressor complex is disassembled and SnRK1 is released to inhibit the activity of TOR (BeldaPalazón et al., 2020). Moreover, there are a number of ABA responsive elements in the promoters of ATGs (Yue et al., 2018), suggesting that the ABA signaling pathway and autophagy can regulate each other alternatively according to environmental conditions to ensure the normal growth of plants.

Ethylene is another stress response phytohormone. It was shown that the ethylene signaling pathway increases ROS levels. It was shown that antioxidant systems can also be activated by ethylene to protect cells from excessive ROS damage (Mersmann et al., 2010; Jiang et al., 2013; Yamauchi et al., 2014). The production of ROS by stress can cause damage to proteins and organelles. These damaged cellular components will then need to be cleared by autophagy (Signorelli et al., 2019). However, the relationship between ethylene signaling and autophagy is still elusive. In the autophagy-deficient mutants atg5 and atg9, a series of ethylene signaling genes are induced (Masclaux-Daubresse et al., 2014). Under drought stress, the transcription factor ERF5 (Ethylene Response Factor 5) binds to the promoters of ATG8 and ATG18 to increase their expression and autophagy (MasclauxDaubresse et al., 2014). More work is still needed to uncover the specific mechanisms of how ethylene signaling affects autophagy. 
Crosstalk between brassinosteroid (BR) signaling and autophagy has recently been revealed. In Arabidopsis, TOR signaling can regulate the protein level of the transcription factor BZR1 (brassinazole-resistant 1), a key regulatory factor in BR signaling, to maintain plant growth and development under carbon starvation. Decreased expression of TOR results in reduced expression of BR-responsive genes and subsequently inhibition of plant growth. This phenotype can be partially recovered by the increase of active forms of BZR1 (Zhang et al., 2016). In addition, Arabidopsis ribosomal protein S6 kinase 2 (S6K2), a direct downstream target of TOR, can directly phosphorylate the BR signaling component BIN2 (brassinosteroid insensitive 2), indicating the TOR-S6K2-BIN2 signaling pathway mediates plant growth (Xiong et al., 2017). Another BR signaling positive regulator, transcription factor BRIEMS SUPPERSSOR 1 (BES1), is selectively degraded through a DOMINANT SUPPRESSOR OF KAR 2 (DSK2)-mediated autophagy pathway under starvation and drought stresses in Arabidopsis (Nolan et al., 2017). BR signaling also regulates autophagy in turn. Under brassinolide treatment, the expression of ATGs and the formation of autophagosomes are reduced in BZR1-silenced tomato plants, but are enhanced in BZR1 overexpressing tomato plants, suggesting feedback regulation of BR signaling on autophagy (Wang et al., 2019). Recently, it has been found that cold and BR can enhance the stability of BZR1 that can bind to the promoter of ATGs and NBR1 to increase the expression of these genes resulting in enhanced autophagy in tomato. The increased autophagy promotes the degradation of damaged insoluble ubiquitinated proteins though selective autophagy receptor NBR1-mediated pathway, indicating that BR positively regulates NBR1-dependent selective autophagy in response to cold (Chi et al., 2020).

Auxin was discovered in maize and in Arabidopsis suspension culture to activate TOR kinase, as evidenced by increased phosphorylation of T449 on S6K1, a direct downstream target of TOR (Beltrán-Peña et al., 2002; Turck et al., 2004; Schepetilnikov et al., 2013). Auxin biosynthesis induced by light and glucose synthesized by photosynthesis activate TOR and promote the growth of shoot meristems in Arabidopsis (Cai et al., 2017; Li et al., 2017). In Arabidopsis, ROP (Rho-like GTPases from plants) small GTPases member, ROP2, directly activates TOR kinase in response to auxin (Li et al., 2017; Schepetilnikov et al., 2017). Furthermore, an E3 ubiquitin ligase COP1 (Constitutive Photomorphogenesis 1) functions as an upstream regulator of ROP2 and negatively regulates auxin-ROP2-TOR signaling pathway in response to light (Cai et al., 2017). Agreeing with auxin's ability to activate TOR, adding exogenous auxin to seedlings under various abiotic stress conditions can inhibit autophagy ( $\mathrm{Pu}$ et al., 2017). In response to glucose stress, Arabidopsis sugar sensor HXK1 (hexokinase 1) and energy sensor KIN10 suppress TOR activity to inhibit constitutive autophagy modulating function of the peroxisomes, which contributes to the production of ROS and IAA to regulate root meristem (Huang et al., 2019a). Induction of auxin signaling can also activate the differentiation of xylem cells. Autophagy is also induced during tracheary element differentiation, although the underlying mechanism is not clear (Ibañes et al., 2009; Kwon et al., 2010). As an activator of TOR, an increase in auxin leads to induction of TOR activity, which occurs simultaneously with the induction of autophagy in the xylem, suggesting that autophagy is activated despite the presence of active TOR (Pu et al., 2017). The BR signaling is required for proper arrangement of the plant-shoot vasculature (Ibañes et al., 2009). In response to BR, autophagy is activated through the activation of RabG3b, a small GTP binding protein, to promote the differentiation of tracheary elements (Kwon et al., 2010). These observations imply that autophagy may be regulated through additional unknown pathways other than the TOR signaling in the xylem.

The effect of phytohormone jasmonic acid (JA) signaling on downstream genes can be affected by TOR inhibition (Dong et al., 2015). JA has been shown to regulate the expression of ERFs, which encode an inducer of resistance to abiotic stresses, and JA-related ERF elements are widely present in the promoters of ATGs, indicating crosstalk between JA signaling and autophagy (Yue et al., 2018; Figure 3). JA is also involved in the response to biotic stress. In Arabidopsis, WRKY33 not only regulates the expression of resistance genes in response to fungal pathogens through JA signaling, but can also interact with ATG18a to regulate autophagy (Lai et al., 2011). High levels of the phytohormone SA can lead to autophagy-related PCD in Arabidopsis, and the induction of PCD during senescence in atg5 is via SA signaling (Yoshimoto et al., 2009). Metabolomic and transcriptomic analyses have shown that SA biosynthesis is up-regulated in Arabidopsis atg5 mutant under low nitrate conditions (Masclaux-Daubresse et al., 2014). It was proposed that a ROS burst is induced by SA accumulation, which in turn promotes autophagy to reduce ROS, indicating negative feedback (Masclaux-Daubresse et al., 2014). However, the regulatory mechanisms connecting SA signaling and autophagy are still unclear.

\section{FUTURE PERSPECTIVES}

The development of a plant from a seed into a mature organism involves the continuous differentiation of new organs and the degeneration of old organs. Autophagy is not only responsible for the removal of cellular waste components, but also plays key regulatory roles in different developmental stages and in response to a wide range of biotic and abiotic environmental stresses. However, excessive autophagy can also damage normal structural components of cells. The level of autophagy is carefully regulated by plants through a variety of pathways according to changing environmental conditions. Environmental signals regulate the initiation of autophagy by affecting TOR kinase activity or though other TOR-independent pathway. Many plant hormones also regulate autophagy, which in turn also provides feedback regulation on these signaling pathways, which jointly ensures the normal growth and development of plants. Recent studies have also deciphered the regulation of autophagosome biogenesis. For example, under ER stress, the phospholipid-binding activity of ATG18a can be activated by reversible persulfidation at Cys103 to negatively regulate autophagosome formation and ensure the appropriate physiological response (Aroca et al., 2021). During 
nutrient starvation, coat protein complex II (COPII) vesicles act as a membrane source for autophagosome formation, mediated by the specific recognition of ATG8e by a COPII component, AtSar1d (Zeng et al., 2021). ROP8, a member of the Rho family guanosine triphosphatases (GTPases) of plants (ROPs), promotes autophagosome formation via its downstream effector Sec5, indicating a previously unappreciated role of ROP8-Sec5 signaling in autophagy promotion (Lin et al., 2021).

Despite the recent achievements uncovering the interactions between signaling pathways and autophagy in plants, there are still many open questions. As an important regulator of autophagy, how does TOR accurately identify different external signals to make the appropriate responses? How are stress signals, ROS signals, and plant hormone signals, which can all regulate autophagy levels, related?How do plants accurately regulate the dynamics of autophagy? The integrated regulatory mechanisms connecting stresses, plant hormones, and autophagy in plants are still unclear. The study of the molecular mechanisms by which

\section{REFERENCES}

Ahn, C. S., Han, J. A., Lee, H. S., Lee, S., and Pai, H. S. (2011). The PP2A regulatory subunit Tap46, a component of the TOR signaling pathway, modulates growth and metabolism in plants. Plant Cell 23, 185-209. doi: 10.1105/tpc.110.074005

Anderson, G. H., Veit, B., and Hanson, M. R. (2005). The Arabidopsis AtRaptor genes are essential for post-embryonic plant growth. BMC Biol. 3:12. doi: 10 . 1186/1741-7007-3-12

Aroca, A., Yruela, I., Gotor, C., and Bassham, D. C. (2021). Persulfidation of ATG18a regulates autophagy under ER stress in Arabidopsis. Proc. Natl. Acad. Sci. USA 118:e2023604118. doi: 10.1073/pnas.2023604118

Avin-Wittenberg, T. (2019). Autophagy and its role in plant abiotic stress management. Plant Cell Environ. 42, 1045-1053. doi: 10.1111/pce.13404

Avin-Wittenberg, T., Bajdzienko, K., Wittenberg, G., Alseekh, S., Tohge, T., Bock, R., et al. (2015). Global analysis of the role of autophagy in cellular metabolism and energy homeostasis in Arabidopsis seedlings under carbon starvation. Plant Cell 27, 306-322. doi: 10.1105/tpc.114.134205

Baena-González, E., and Hanson, J. (2017). Shaping plant development through the SnRK1-TOR metabolic regulators. Curr. Opin. Plant Biol. 35, 152-157. doi: 10.1016/j.pbi.2016.12.004

Bao, Y., Song, W. M., Wang, P., Yu, X., Li, B., Jiang, C., et al. (2020). COST1 regulates autophagy to control plant drought tolerance. Proc. Natl. Acad. Sci. USA 117, 7482-7493. doi: 10.1073/pnas.1918539117

Belda-Palazón, B., Adamo, M., Valerio, C., Ferreira, L. J., Confraria, A., Reis-Barata, D., et al. (2020). A dual function of SnRK2 kinases in the regulation of SnRK1 and plant growth. Nat. Plants 6, 1345-1353. doi: 10.1038/s41477-020-00778-w

Beltrán-Peña, E., Aguilar, R., Ortíz-López, A., Dinkova, T. D., and De Jiménez, E. S. (2002). Auxin stimulates S6 ribosomal protein phosphorylation in maize thereby affecting protein synthesis regulation. Physiol. Plant. 115, 291-297. doi: 10.1034/j.1399-3054.2002.1150216.X

Cai, W., Li, X., Liu, Y., Wang, Y., Zhou, Y., Xu, T., et al. (2017). COP1 integrates light signals to ROP2 for cell cycle activation. Plant Signal Behav. 12:e1363946. doi: 10.1080/15592324.2017.1363946

Chen, J. G., and Jones, A. M. (2004). AtRGS1 function in Arabidopsis thaliana. Methods Enzymol. 389, 338-350. doi: 10.1016/s0076-6879(04)89020-7

Chen, L., Su, Z. Z., Huang, L., Xia, F. N., Qi, H., Xie, L. J., et al. (2017). The AMP-activated protein kinase KIN10 is involved in the regulation of autophagy in Arabidopsis. Front. Plant Sci. 8:1201. doi: 10.3389/fpls.2017.0 1201

Chi, C., Li, X., Fang, P., Xia, X., Shi, K., Zhou, Y., et al. (2020). Brassinosteroids act as a positive regulator of NBR1-dependent selective autophagy in response to chilling stress in tomato. J. Exp. Bot. 71, 1092-1106. doi: 10.1093/jxb/erz466

Chung, T. (2019). How phosphoinositides shape autophagy in plant cells. Plant Sci. 281, 146-158. doi: 10.1016/j.plantsci.2019.01.017 environmental signals regulate autophagy is crucial to uncover these mysteries.

\section{AUTHOR CONTRIBUTIONS}

PW, TW, TS, and CM conceived and wrote the review. JH, ML, and YZ contributed to revision of the manuscript. All authors have read and agreed to the published version of the manuscript.

\section{FUNDING}

This review was funded by the National Natural Science Foundation of China (31770290 and 31970301), the Agricultural Variety Improvement Project of Shandong Province (2019LZGC015), and the China Postdoctoral Science Foundation (2017M612333).

Chung, T., Phillips, A. R., and Vierstra, R. D. (2010). ATG8 lipidation and ATG8-mediated autophagy in Arabidopsis require ATG12 expressed from the differentially controlled ATG12A and ATG12B loci. Plant J. 62, 483-493.

Deprost, D., Truong, H. N., Robaglia, C., and Meyer, C. (2005). An Arabidopsis homolog of RAPTOR/KOG1 is essential for early embryo development. Biochem. Biophys. Res. Commun. 326, 844-850. doi: 10.1016/j.bbrc.2004.11.117

Deretic, V. (2012). Autophagy as an innate immunity paradigm: expanding the scope and repertoire of pattern recognition receptors. Curr. Opin. Immunol. 24, 21-31. doi: 10.1016/j.coi.2011.10.006

Díaz-Troya, S., Pérez-Pérez, M. E., Florencio, F. J., and Crespo, J. L. (2008). The role of TOR in autophagy regulation from yeast to plants and mammals. Autophagy 4, 851-865. doi: 10.4161/auto.6555

Dobrenel, T., Caldana, C., Hanson, J., Robaglia, C., Vincentz, M., Veit, B., et al. (2016). TOR signaling and nutrient sensing. Ann. Rev. Plant Biol. 67, 261-285.

Dong, P., Xiong, F., Que, Y., Wang, K., Yu, L., Li, Z., et al. (2015). Expression profiling and functional analysis reveals that TOR is a key player in regulating photosynthesis and phytohormone signaling pathways in Arabidopsis. Front. Plant Sci. 6:677. doi: 10.3389/fpls.2015.00677

Dong, Y., Silbermann, M., Speiser, A., Forieri, I., Linster, E., Poschet, G., et al. (2017). Sulfur availability regulates plant growth via glucose-TOR signaling. Nat. Commun. 8:1174.

Dündar, G., Shao, Z., Higashitani, N., Kikuta, M., Izumi, M., and Higashitani, A. (2019). Autophagy mitigates high-temperature injury in pollen development of Arabidopsis thaliana. Dev. Biol. 456, 190-200. doi: 10.1016/j.ydbio.2019. 08.018

Fu, L., Wang, P., and Xiong, Y. (2020). Target of rapamycin signaling in plant stress responses. Plant Physiol. 182, 1613-1623. doi: 10.1104/pp.19.01214

Gong, W., Li, B., Zhang, B., and Chen, W. (2020). ATG4 mediated Psm ES4326/AvrRpt2-induced autophagy dependent on salicylic acid in Arabidopsis Thaliana. Int. J. Mol. Sci. 21:5147. doi: 10.3390/ijms21145147

Gou, W., Li, X., Guo, S., Liu, Y., Li, F., and Xie, Q. (2019). Autophagy in plant: a new orchestrator in the regulation of the phytohormones homeostasis. Int. J. Mol. Sci. 20:2900. doi: 10.3390/ijms20122900

Guiboileau, A., Avila-Ospina, L., Yoshimoto, K., Soulay, F., Azzopardi, M., Marmagne, A., et al. (2013). Physiological and metabolic consequences of autophagy deficiency for the management of nitrogen and protein resources in Arabidopsis leaves depending on nitrate availability. New Phytol. 199, 683-694. doi: 10.1111/nph.12307

Guiboileau, A., Yoshimoto, K., Soulay, F., Bataillé, M. P., Avice, J. C., and MasclauxDaubresse, C. (2012). Autophagy machinery controls nitrogen remobilization at the whole-plant level under both limiting and ample nitrate conditions in Arabidopsis. New Phytol. 194, 732-740. doi: 10.1111/j.1469-8137.2012. 04084.x 
Guillaumot, D., Guillon, S., Morsomme, P., and Batoko, H. (2009). ABA, porphyrins and plant TSPO-related protein. Plant Signal Behav. 4, 1087-1090. doi: $10.4161 /$ psb.4.11.9796

Hachez, C., Veljanovski, V., Reinhardt, H., Guillaumot, D., Vanhee, C., Chaumont, F., et al. (2014). The Arabidopsis abiotic stress-induced TSPOrelated protein reduces cell-surface expression of the aquaporin PIP2;7 through protein-protein interactions and autophagic degradation. Plant Cell 26, 4974-4990. doi: 10.1105/tpc.114.134080

Hanaoka, H., Noda, T., Shirano, Y., Kato, T., Hayashi, H., Shibata, D., et al. (2002). Leaf senescence and starvation-induced chlorosis are accelerated by the disruption of an Arabidopsis autophagy gene. Plant Physiol. 129, 1181-1193. doi: 10.1104/pp.011024

Hatsugai, N., Kuroyanagi, M., Yamada, K., Meshi, T., Tsuda, S., Kondo, M., et al. (2004). A plant vacuolar protease. VPE, mediates virus-induced hypersensitive cell death. Science 305, 855-858. doi: 10.1126/science.1099859

Hoehenwarter, W., Mönchgesang, S., Neumann, S., Majovsky, P., Abel, S., and Müller, J. (2016). Comparative expression profiling reveals a role of the root apoplast in local phosphate response. BMC Plant Biol. 16:106. doi: 10.1186/ s12870-016-0790-8

Hofius, D., Schultz-Larsen, T., Joensen, J., Tsitsigiannis, D. I., Petersen, N. H., Mattsson, O., et al. (2009). Autophagic components contribute to hypersensitive cell death in Arabidopsis. Cell 137, 773-783. doi: 10.1016/s9999-9994(09) 00527-3

Honig, A., Avin-Wittenberg, T., Ufaz, S., and Galili, G. (2012). A new type of compartment, defined by plant-specific Atg8-interacting proteins, is induced upon exposure of Arabidopsis plants to carbon starvation. Plant Cell 24, 288-303. doi: 10.1105/tpc.111.093112

Huang, L., Yu, L. J., Zhang, X., Fan, B., Wang, F. Z., Dai, Y. S., et al. (2019a). Autophagy regulates glucose-mediated root meristem activity by modulating ROS production in Arabidopsis. Autophagy 15, 407-422. doi: 10.1080/ 15548627.2018.1520547

Huang, X., Zheng, C., Liu, F., Yang, C., Zheng, P., Lu, X., et al. (2019b). Genetic analyses of the Arabidopsis ATG1 kinase complex reveal both kinase-dependent and independent autophagic routes during fixed-carbon starvation. Plant Cell 31, 2973-2995. doi: 10.1105/tpc. 19.00066

Ibañes, M., Fàbregas, N., Chory, J., and Caño-Delgado, A. I. (2009). Brassinosteroid signaling and auxin transport are required to establish the periodic pattern of Arabidopsis shoot vascular bundles. Proc. Natl. Acad. Sci. USA 106, 1363013635. doi: 10.1073/pnas.0906416106

Izumi, M., Hidema, J., Makino, A., and Ishida, H. (2013). Autophagy contributes to nighttime energy availability for growth in Arabidopsis. Plant physiol. 161, 1682-1693. doi: 10.1104/pp.113.215632

Jiang, C., Belfield, E. J., Cao, Y., Smith, J. A., and Harberd, N. P. (2013). An Arabidopsis soil-salinity-tolerance mutation confers ethylene-mediated enhancement of sodium/potassium homeostasis. Plant Cell 25, 3535-3552. doi: 10.1105/tpc.113.115659

Jiang, L., Lu, Y., Zheng, X., Yang, X., Chen, Y., Zhang, T., et al. (2021a). The plant protein NbP3IP directs degradation of Rice stripe virus p3 silencing suppressor protein to limit virus infection through interaction with the autophagy-related protein NbATG8. New Phytol. 229, 1036-1051. doi: 10.1111/nph.16917

Jiang, L., Zheng, X., Liu, Y., Chen, J., Lu, Y., and Yan, F. (2021b). Plant protein P3IP participates in the regulation of autophagy in Nicotiana benthamiana. Plant Signal Behav. 16:1861768. doi: 10.1080/15592324.2020.1861768

Jiao, Y., Srba, M., Wang, J., and Chen, W. (2019). Correlation of autophagosome formation with degradation and endocytosis Arabidopsis regulator of G-protein signaling (RGS1) through ATG8a. Int. J. Mol. Sci. 20:4190. doi: 10.3390/ ijms 20174190

Jung, H., Lee, H. N., Marshall, R. S., Lomax, A. W., Yoon, M. J., Kim, J., et al. (2020). Arabidopsis cargo receptor NBR1 mediates selective autophagy of defective proteins. J. Exp. Bot. 71, 73-89. doi: 10.1093/jxb/erz404

Kang, S., Shin, K. D., Kim, J. H., and Chung, T. (2018). Autophagy-related (ATG) 11. ATG9 and the phosphatidylinositol 3-kinase control ATG2-mediated formation of autophagosomes in Arabidopsis. Plant Cell Rep. 37, 653-664. doi: 10.1007/s00299-018-2258-9

Ketelaar, T., Voss, C., Dimmock, S. A., Thumm, M., and Hussey, P. J. (2004). Arabidopsis homologues of the autophagy protein Atg8 are a novel family of microtubule binding proteins. FEBS Lett. 567, 302-306. doi: 10.1016/j.febslet. 2004.04.088
Klionsky, D. J. (2008). Autophagy revisited: a conversation with Christian de Duve. Autophagy 4, 740-743. doi: 10.4161/auto.6398

Kravchenko, A., Citerne, S., Jéhanno, I., Bersimbaev, R. I., Veit, B., Meyer, C., et al. (2015). Mutations in the Arabidopsis Lst8 and Raptor genes encoding partners of the TOR complex, or inhibition of TOR activity decrease abscisic acid (ABA) synthesis. Biochem. Biophys. Res. Commun. 467, 992-997. doi: 10.1016/j.bbrc.2015.10.028

Kwon, S. I., Cho, H. J., Jung, J. H., Yoshimoto, K., Shirasu, K., and Park, O. K. (2010). The Rab GTPase RabG3b functions in autophagy and contributes to tracheary element differentiation in Arabidopsis. Plant J. 64, 151-164.

Lai, Z., Wang, F., Zheng, Z., Fan, B., and Chen, Z. (2011). A critical role of autophagy in plant resistance to necrotrophic fungal pathogens. Plant J. 66, 953-968. doi: 10.1111/j.1365-313x.2011.04553.x

Le Bars, R., Marion, J., Le Borgne, R., Satiat-Jeunemaitre, B., and Bianchi, M. W. (2014). ATG5 defines a phagophore domain connected to the endoplasmic reticulum during autophagosome formation in plants. Nat. Commun. 5:4121.

Li, F., and Vierstra, R. D. (2014). Arabidopsis ATG11, a scaffold that links the ATG1-ATG13 kinase complex to general autophagy and selective mitophagy. Autophagy 10, 1466-1467. doi: 10.4161/auto.29320

Li, X., Cai, W., Liu, Y., Li, H., Fu, L., Liu, Z., et al. (2017). Differential TOR activation and cell proliferation in Arabidopsis root and shoot apexes. Proc. Natl. Acad. Sci. USA. 114, 2765-2770. doi: 10.1073/pnas.1618782114

Lin, Y., Zeng, Y., Zhu, Y., Shen, J., Ye, H., and Jiang, L. (2021). Plant Rho GTPase signaling promotes autophagy. Mol. Plant 14, 905-920. doi: 10.1016/j.molp. 2021.03.021

Liu, Y., and Bassham, D. C. (2012). Autophagy: pathways for self-eating in plant cells. Annu. Rev. Plant Biol. 63, 215-237. doi: 10.1146/annurev-arplant042811-105441

Liu, Y., Schiff, M., Czymmek, K., Tallóczy, Z., Levine, B., and Dinesh-Kumar, S. P. (2005). Autophagy regulates programmed cell death during the plant innate immune response. Cell 121, 567-577. doi: 10.1016/j.cell.2005.03.007

Mahfouz, M. M., Kim, S., Delauney, A. J., and Verma, D. P. (2006). Arabidopsis TARGET OF RAPAMYCIN interacts with RAPTOR, which regulates the activity of S6 kinase in response to osmotic stress signals. Plant Cell 18, 477-490. doi: $10.1105 /$ tpc. 105.035931

Marshall, R. S., and Vierstra, R. D. (2018). Autophagy: the master of bulk and selective recycling. Annu. Rev. Plant Biol. 69, 173-208. doi: 10.1146/annurevarplant-042817-040606

Masclaux-Daubresse, C., Clément, G., Anne, P., Routaboul, J. M., Guiboileau, A., Soulay, F., et al. (2014). Stitching together the multiple dimensions of autophagy using metabolomics and transcriptomics reveals impacts on metabolism, development, and plant responses to the environment in Arabidopsis. Plant Cell 26, 1857-1877. doi: 10.1105/tpc.114.124677

Máthé, C., M-Hamvas, M., Freytag, C., and Garda, T. (2021). The protein phosphatase PP2A plays multiple roles in plant development by regulation of vesicle traffic-facts and questions. Int. J. Mol. Sci. 22:975. doi: 10.3390/ ijms 22020975

Matsuura, A., Tsukada, M., Wada, Y., and Ohsumi, Y. (1997). Apg1p, a novel protein kinase required for the autophagic process in Saccharomyces cerevisiae. Gene 192, 245-250. doi: 10.1016/s0378-1119(97)00084-x

Mersmann, S., Bourdais, G., Rietz, S., and Robatzek, S. (2010). Ethylene signaling regulates accumulation of the FLS2 receptor and is required for the oxidative burst contributing to plant immunity. Plant Physiol. 154, 391-400. doi: 10. 1104/pp.110.154567

Michaeli, S., Honig, A., Levanony, H., Peled-Zehavi, H., and Galili, G. (2014). Arabidopsis ATG8-INTERACTING PROTEIN1 is involved in autophagydependent vesicular trafficking of plastid proteins to the vacuole. Plant Cell 26, 4084-4101. doi: 10.1105/tpc.114.129999

Minina, E. A., Moschou, P. N., Vetukuri, R. R., Sanchez-Vera, V., Cardoso, C., Liu, Q., et al. (2018). Transcriptional stimulation of rate-limiting components of the autophagic pathway improves plant fitness. J. Exp. Bot. 69, 1415-1432. doi: $10.1093 /$ jxb/ery010

Moreau, M., Azzopardi, M., Clément, G., Dobrenel, T., Marchive, C., Renne, C., et al. (2012). Mutations in the Arabidopsis homolog of LST8/GßL, a partner of the target of Rapamycin kinase, impair plant growth, flowering, and metabolic adaptation to long days. Plant Cell 24, 463-481. doi: 10.1105/tpc.111.09 1306 
Mugume, Y., Kazibwe, Z., and Bassham, D. C. (2020). Target of Rapamycin in control of autophagy: puppet master and signal integrator. Int. J. Mol. Sci. 21:8259. doi: $10.3390 /$ ijms 21218259

Müller, J., Toev, T., Heisters, M., Teller, J., Moore, K. L., Hause, G., et al. (2015). Iron-dependent callose deposition adjusts root meristem maintenance to phosphate availability. Dev. Cell 33, 216-230. doi: 10.1016/j.devcel.2015. 02.007

Naumann, C., Müller, J., Sakhonwasee, S., Wieghaus, A., Hause, G., Heisters, M., et al. (2019). The local phosphate deficiency response activates endoplasmic reticulum stress-dependent autophagy. Plant physiol. 179, 460-476. doi: 10. 1104/pp.18.01379

Nolan, T. M., Brennan, B., Yang, M., Chen, J., Zhang, M., Li, Z., et al. (2017). Selective autophagy of BES1 mediated by DSK2 balances plant growth and survival. Dev. Cell 41, 33-46. doi: 10.1016/j.devcel.2017.03.013

Nukarinen, E., Nägele, T., Pedrotti, L., Wurzinger, B., Mair, A., Landgraf, R., et al. (2016). Quantitative phosphoproteomics reveals the role of the AMPK plant ortholog SnRK1 as a metabolic master regulator under energy deprivation. Sci. Rep. 6:31697.

Ohsumi, Y. (2001). Molecular dissection of autophagy: two ubiquitin-like systems. Nat. Rev. Mol. Cell Biol. 2, 211-216. doi: 10.1038/35056522

Peng, Y., van Wersch, R., and Zhang, Y. (2018). Convergent and divergent signaling in PAMP-triggered immunity and effector-triggered immunity. Mol. Plant Microbe. Interact. 31, 403-409. doi: 10.1094/mpmi-06-17-0145-cr

Popa, C., Li, L., Gil, S., Tatjer, L., Hashii, K., Tabuchi, M., et al. (2016). The effector AWR5 from the plant pathogen Ralstonia solanacearum is an inhibitor of the TOR signalling pathway. Sci. Rep. 10:20947.

$\mathrm{Pu}, \mathrm{Y}$., Luo, X., and Bassham, D. C. (2017). TOR-dependent and -independent pathways regulate autophagy in Arabidopsis thaliana. Front. Plant Sci. $8: 1204$.

Qi, H., Li, J., Xia, F. N., Chen, J. Y., Lei, X., Han, M. Q., et al. (2019). Arabidopsis SINAT proteins control autophagy by mediating ubiquitylation and degradation of ATG13. Plant Cell 32, 263-284. doi: 10.1105/tpc.19.00413

Raghavendra, A. S., Gonugunta, V. K., Christmann, A., and Grill, E. (2010). ABA perception and signalling. Trends Plant Sci. 15, 395-401. doi: 10.1016/j.tplants. 2010.04.006

Sankaranarayanan, S., and Samuel, M. A. (2015). A proposed role for selective autophagy in regulating auxin-dependent lateral root development under phosphate starvation in Arabidopsis. Plant Signal. Behav. 10:e989749. doi: 10.4161/15592324.2014.989749

Schepetilnikov, M., Dimitrova, M., Mancera-Martínez, E., Geldreich, A., Keller, M., and Ryabova, L. A. (2013). TOR and S6K1 promote translation reinitiation of uORF-containing mRNAs via phosphorylation of eIF3h. EMBO J. 32, 10871102. doi: 10.1038/emboj.2013.61

Schepetilnikov, M., Makarian, J., Srour, O., Geldreich, A., Yang, Z., Chicher, J., et al. (2017). GTPase ROP2 binds and promotes activation of target of rapamycin. TOR, in response to auxin. EMBO J. 36, 886-903. doi: $10.15252 /$ embj.201694816

Sedaghatmehr, M., Thirumalaikumar, V. P., Kamranfar, I., Marmagne, A., Masclaux-Daubresse, C., and Balazadeh, S. (2019). A regulatory role of autophagy for resetting the memory of heat stress in plants. Plant Cell Environ. 42, 1054-1064. doi: 10.1111/pce.13426

Shemi, A., Ben-Dor, S., and Vardi, A. (2015). Elucidating the composition and conservation of the autophagy pathway in photosynthetic eukaryotes. Autophagy 11, 701-715. doi: 10.1080/15548627.2015.1034407

Signorelli, S., Tarkowski, T. P., Van den Ende, W., and Bassham, D. C. (2019). Linking autophagy to abiotic and biotic stress responses. Trends Plant Sci. 24, 413-430. doi: 10.1016/j.tplants.2019.02.001

Slavikova, S., Ufaz, S., Avin-Wittenberg, T., Levanony, H., and Galili, G. (2008). An autophagy-associated Atg8 protein is involved in the responses of Arabidopsis seedlings to hormonal controls and abiotic stresses. J. Exp. Bot. 59, 4029-4043. doi: $10.1093 / \mathrm{jxb} / \mathrm{ern} 244$

Son, O., Kim, S., Kim, D., Hur, Y. S., Kim, J., and Cheon, C. I. (2018). Involvement of TOR signaling motif in the regulation of plant autophagy. B Biochem. Biophys. Res. Commun. 501, 643-647. doi: 10.1016/j.bbrc.2018.05.027

Sørensen, D. M., Holen, H. W., Holemans, T., Vangheluwe, P., and Palmgren, M. G. (2015). Towards defining the substrate of orphan P5A-ATPases. Biochim. Biophys. Acta 1850, 524-535. doi: 10.1016/j.bbagen.2014.05.008
Soto-Burgos, J., and Bassham, D. C. (2017). SnRK1 activates autophagy via the TOR signaling pathway in Arabidopsis thaliana. PLoS One 12:e0182591. doi: 10.1371/journal.pone.0182591

Suttangkakul, A., Li, F., Chung, T., and Vierstra, R. D. (2011). The ATG1/ATG13 protein kinase complex is both a regulator and a target of autophagic recycling in Arabidopsis. Plant Cell 23, 3761-3779. doi: 10.1105/tpc.111.09 0993

Takahashi, H., Kopriva, S., Giordano, M., Saito, K., and Hell, R. (2011). Sulfur assimilation in photosynthetic organisms: molecular functions and regulations of transporters and assimilatory enzymes. Annu. Rev. Plant Biol. 62, 157-184. doi: 10.1146/annurev-arplant-042110-103921

Teh, O. K., and Hofius, D. (2014). Membrane trafficking and autophagy in pathogen-triggered cell death and immunity. J. Exp. Bot. 65, 1297-1312. doi: $10.1093 / \mathrm{jxb} / \mathrm{ert} 441$

Thirumalaikumar, V. P., Wagner, M., Balazadeh, S., and Skirycz, A. (2021). Autophagy is responsible for the accumulation of proteogenic dipeptides in response to heat stress in Arabidopsis thaliana. FEBS J. 288, 281-292. doi: 10.1111/febs. 15336

Thompson, A. R., Doelling, J. H., Suttangkakul, A., and Vierstra, R. D. (2005). Autophagic nutrient recycling in Arabidopsis directed by the ATG8 and ATG12 conjugation pathways. Plant physiol. 138, 2097-2110. doi: 10.1104/pp.105. 060673

Ticconi, C. A., Lucero, R. D., Sakhonwasee, S., Adamson, A. W., Creff, A., Nussaume, L., et al. (2009). ER-resident proteins PDR2 and LPR1 mediate the developmental response of root meristems to phosphate availability. Proc. Natl. Acad. Sci. USA 106, 14174-14179. doi: 10.1073/pnas.0901778106

Tong, X., Liu, S. Y., Zou, J. Z., Zhao, J. J., Zhu, F. F., Chai, L. X., et al. (2021). A small peptide inhibits siRNA amplification in plants by mediating autophagic degradation of SGS3/RDR6 bodies. EMBO J. 40:e108050.

Tsai, A. Y., and Gazzarrini, S. (2014). Trehalose-6-phosphate and SnRK1 kinases in plant development and signaling: the emerging picture. Front. Plant Sci. 5:119. doi: $10.3389 /$ fpls.2014.00119

Turck, F., Zilbermann, F., Kozma, S. C., Thomas, G., and Nagy, F. (2004). Phytohormones participate in an S6 kinase signal transduction pathway in Arabidopsis. Plant Physiol. 134, 1527-1535. doi: 10.1104/pp.103.035873

Üstün, S., Hafrén, A., Liu, Q., Marshall, R. S., Minina, E. A., Bozhkov, P. V., et al. (2018). Bacteria exploit autophagy for proteasome degradation and enhanced virulence in plants. Plant Cell 30, 668-685. doi: 10.1105/tpc.17.00815

Van Doorn, W. G., and Papini, A. (2013). Ultrastructure of autophagy in plant cells. Autophagy 9, 1922-1936. doi: 10.4161/auto.26275

Van Leene, J., Han, C., Gadeyne, A., Eeckhout, D., Matthijs, C., Cannoot, B., et al. (2019). Capturing the phosphorylation and protein interaction landscape of the plant TOR kinase. Nat. Plants 5, 316-327. doi: 10.1038/s41477-019-0378-z

Vanhee, C., Zapotoczny, G., Masquelier, D., Ghislain, M., and Batoko, H. (2011). The Arabidopsis multistress regulator TSPO is a heme binding membrane protein and a potential scavenger of porphyrins via an autophagy-dependent degradation mechanism. Plant Cell 23, 785-805. doi: 10.1105/tpc. 110.081570

Wang, P., Mugume, Y., and Bassham, D. C. (2018a). New advances in autophagy in plants: regulation, selectivity and function. Semin. Cell Dev. Biol. 80, 113-122. doi: 10.1016/j.semcdb.2017.07.018

Wang, P., Zhao, Y., Li, Z., Hsu, C. C., Liu, X., Fu, L., et al. (2018b). Reciprocal regulation of the TOR kinase and $\mathrm{ABA}$ receptor balances plant growth and stress response. Mol. Cell 69, 100-112. doi: 10.1016/j.molcel.2017.12.002

Wang, Y., Cao, J. J., Wang, K. X., Xia, X. J., Shi, K., Zhou, Y. H., et al. (2019). BZR1 mediates brassinosteroid-induced autophagy and nitrogen starvation in tomato. Plant Physiol. 179, 671-685. doi: 10.1104/pp.18.01028

Wen, X., and Klionsky, D. J. (2016). An overview of macroautophagy in yeast. J. Mol. Biol. 428, 1681-1699. doi: 10.1016/j.jmb.2016.02.021

Woo, J., Park, E., and Dinesh-Kumar, S. P. (2014). Differential processing of Arabidopsis ubiquitin-like Atg8 autophagy proteins by Atg 4 cysteine proteases. Proc. Natl. Acad. Sci. USA 111, 863-868. doi: 10.1073/pnas.1318207111

Wu, J., Michaeli, S., Picchianti, L., Dagdas, Y., Galili, G., and Peled-Zehavi, H. (2021). ATI1 (ATG8-interacting protein 1) and ATI2 define a plant starvationinduced reticulophagy pathway and serve as MSBP1/MAPR5 cargo receptors. Autophagy 25, 1-14. doi: 10.1080/15548627.2021.1872886

Xiong, F., Zhang, R., Meng, Z., Deng, K., Que, Y., Zhuo, F., et al. (2017). Brassinosteriod Insensitive 2 (BIN2) acts as a downstream effector of the Target 
of Rapamycin (TOR) signaling pathway to regulate photoautotrophic growth in Arabidopsis. New Phytol. 213, 233-249. doi: 10.1111/nph.14118

Xiong, Y., Contento, A. L., and Bassham, D. C. (2005). AtATG18a is required for the formation of autophagosomes during nutrient stress and senescence in Arabidopsis thaliana. Plant J. 42, 535-546. doi: 10.1111/j.1365-313x.2005. 02397.x

Yamamoto, H., Kakuta, S., Watanabe, T. M., Kitamura, A., Sekito, T., KondoKakuta, C., et al. (2012). Atg9 vesicles are an important membrane source during early steps of autophagosome formation. J. Cell Biol. 198, 219-233. doi: $10.1083 /$ jcb.201202061

Yamauchi, T., Watanabe, K., Fukazawa, A., Mori, H., Abe, F., Kawaguchi, K., et al. (2014). Ethylene and reactive oxygen species are involved in root aerenchyma formation and adaptation of wheat seedlings to oxygen-deficient conditions. J. Exp. Bot. 65, 261-273. doi: 10.1093/jxb/ert371

Yan, Q., Wang, J., Fu, Z. Q., and Chen, W. (2017). Endocytosis of AtRGS1 is regulated by the autophagy pathway after D-glucose stimulation. Front. Plant Sci. 8:1229. doi: 10.3389/fpls.2017.01229

Yang, M., Zhang, Y., Xie, X., Yue, N., Li, J., Wang, X. B., et al. (2018). Barley stripe mosaic virus $\gamma \mathrm{b}$ protein subverts autophagy to promote viral infection by disrupting the ATG7-ATG8 interaction. Plant Cell 30, 1582-1595. doi: $10.1105 /$ tpc. 18.00122

Yoshida, T., Mogami, J., and Yamaguchi-Shinozaki, K. (2014). ABA-dependent and ABA-independent signaling in response to osmotic stress in plants. Curr. Opin. Plant Biol. 21, 133-139. doi: 10.1016/j.pbi.2014.07.009

Yoshimoto, K., Hanaoka, H., Sato, S., Kato, T., Tabata, S., Noda, T., et al. (2004). Processing of ATG8s, ubiquitin-like proteins, and their deconjugation by ATG4s are essential for plant autophagy. Plant Cell 16, 2967-2983. doi: 10.1105/tpc.104.025395

Yoshimoto, K., Jikumaru, Y., Kamiya, Y., Kusano, M., Consonni, C., Panstruga, R., et al. (2009). Autophagy negatively regulates cell death by controlling NPR1dependent salicylic acid signaling during senescence and the innate immune response in Arabidopsis. Plant Cell 21, 2914-2927.

Yue, W., Nie, X., Cui, L., Zhi, Y., Zhang, T., Du, X., et al. (2018). Genome-wide sequence and expressional analysis of autophagy gene family in bread wheat (Triticum aestivum L.). J. Plant Physiol. 229, 7-21. doi: 10.1016/j.jplph.2018. 06.012
Zeng, Y., Li, B., Ji, C., Feng, L., Niu, F., Deng, C., et al. (2021). A unique AtSar1DAtRabD2a nexus modulates autophagosome biogenesis in Arabidopsis thaliana. Proc. Natl. Acad. Sci. USA 118, e2021293118. doi: 10.1073/pnas.2021293118

Zhang, Z., Zhu, J. Y., Roh, J., Marchive, C., Kim, S. K., Meyer, C., et al. (2016). TOR signaling promotes accumulation of BZR1 to balance growth with carbon availability in Arabidopsis. Curr. Biol. 26, 1854-1860. doi: 10.1016/j.cub.2016. 05.005

Zhou, J., Yu, J. Q., and Chen, Z. (2014). The perplexing role of autophagy in plant innate immune responses. Mol. Plant Pathol. 15, 637-645. doi: 10.1111/mpp. 12118

Zhu, J. K. (2016). Abiotic stress signaling and responses in plants. Cell 167, 313-324. doi: 10.1016/j.cell.2016.08.029

Zhuang, X., Chung, K. P., Cui, Y., Lin, W., Gao, C., Kang, B. H., et al. (2017). ATG9 regulates autophagosome progression from the endoplasmic reticulum in Arabidopsis. Proc. Natl. Acad. Sci. USA 114, E426-E435.

Zhuang, X., Wang, H., Lam, S. K., Gao, C., Wang, X., Cai, Y., et al. (2013). A BAR-domain protein SH3P2, which binds to phosphatidylinositol 3-phosphate and ATG8, regulates autophagosome formation in Arabidopsis. Plant Cell 25, 4596-4615. doi: 10.1105/tpc.113.118307

Conflict of Interest: The authors declare that the research was conducted in the absence of any commercial or financial relationships that could be construed as a potential conflict of interest.

Publisher's Note: All claims expressed in this article are solely those of the authors and do not necessarily represent those of their affiliated organizations, or those of the publisher, the editors and the reviewers. Any product that may be evaluated in this article, or claim that may be made by its manufacturer, is not guaranteed or endorsed by the publisher.

Copyright (c) 2021 Wang, Wang, Han, Li, Zhao, Su and Ma. This is an open-access article distributed under the terms of the Creative Commons Attribution License (CC BY). The use, distribution or reproduction in other forums is permitted, provided the original author(s) and the copyright owner(s) are credited and that the original publication in this journal is cited, in accordance with accepted academic practice. No use, distribution or reproduction is permitted which does not comply with these terms. 